\title{
Can computed tomography be a primary tool for COVID-19 detection? Evidence appraisal through meta-analysis
}

\author{
Edward Pei-Chuan Huang ${ }^{1,2+}$, Chih-Wei Sung ${ }^{2+}$, Chi-Hsin Chen², Cheng-Yi Fann ${ }^{2,3}$, Pei-Chun Lai ${ }^{4,5,6^{*}+}$ and \\ Yen-Ta Huang ${ }^{7,8,9^{*}+}$ (1)
}

Keywords: Computed tomography, COVID-19, Meta-analysis, Likelihood ratio, Sensitivity, Specificity

The World Health Organization (WHO) has officially declared the pandemic of coronavirus disease 2019 (COVID-19) on March 11, 2020 [1]. The current pandemic COVID-19 causes suspicious cases flocking into hospitals. The detection of COVID-19 by traditional reverse-transcription diagnostic polymerase chain reaction (RT-PCR) tests is time-consuming and depends on the reliability of laboratory techniques. Several PCRbased rapid tests have been recently approved and only require less than $30 \mathrm{~min}$. Chest computed tomography (CT) has been suggested as an alternative and reliable tool for the detection of COVID-19 in symptomatic patients in China [2]. However, the American College of Radiology recommended against the use of $\mathrm{CT}$ as a firstline test to diagnose COVID-19 on March 11, 2020 [3]. To validate this recommendation, we performed a systematic review with meta-analysis to evaluate the diagnostic value of chest CT in COVID-19.

Two investigators independently searched with the term of "novel coronavirus" or "coronavirus disease 2019" or "COVID-19" or "SARS-CoV-2" combined with

\footnotetext{
* Correspondence: debbie0613.lai@gmail.com; uncleda.haung@gmail.com ${ }^{\dagger}$ Edward Pei-Chuan Huang, Chih-Wei Sung, Pei-Chun Lai and Yen-Ta Huang contributed equally to this work.

${ }^{4}$ Evidence-based Medicine Center, Department of Medical Education, Hualien Tzu Chi Hospital, Buddhist Tzu Chi Medical Foundation, 707, Sec. 3,

Chung-Yang Rd, Hualien 970, Taiwan

${ }^{7}$ Division of Experimental Surgery, Department of Surgery, Hualien Tzu Chi Hospital, Buddhist Tzu Chi Medical Foundation, 707, Sec. 3, Chung-Yang Rd, Hualien 970, Taiwan

Full list of author information is available at the end of the article
}

"computed tomography" or "CT" on PubMed, Web of Science, Embase, Cochrane Library, and China Academic Journals Full-text Database (CJFD) till March 13, 2020. Studies were excluded due to duplication, irrelevant topics, case report(s) or series, availability of only the abstract, and insufficient data. Two investigators independently extracted data for pooled estimates of sensitivity, specificity, and positive and negative likelihood ratio $[\mathrm{LR}(+)$ and $L R(-)]$ with $95 \%$ confidence intervals (CIs) calculated by midas command in Stata 15 (StataCorp LLC., College Station, TX, USA). Heterogeneity across studies was examined using $I^{2}$. Fagan's Nomogram plot analysis was performed to compare the pretest probability, the LR, and the post-test probability.

Only 4 studies screened from 372 relevant articles were eligible [2, 4-6]. A total of 1286 patients in China were screened for COVID-19 using both RT-PCR and chest CT. The pooled sensitivity and specificity of chest CT were 0.95 (95\% CI, 0.93-0.97) and 0.09 (95\% CI, $0.02-0.34)$, respectively, using RT-PCR as the reference method (Fig. 1). The pooled LR (+) and LR (-) of chest CT were as low as 1.10 (95\% CI, 0.90-1.20) and 0.49 (95\% CI, 0.10-2.33), respectively. We further used Fagan's Nomogram to calculate the post-test probability of diagnosed COVID-19 by chest CT (Fig. 2). Our analysis revealed that, regardless the levels of pre-test probabilities $(25,50$, and $75 \%)$, the post-test probabilities were only slightly changed.

Our results indicate a high sensitivity of chest CT for the detection of COVID-19. However, our results 


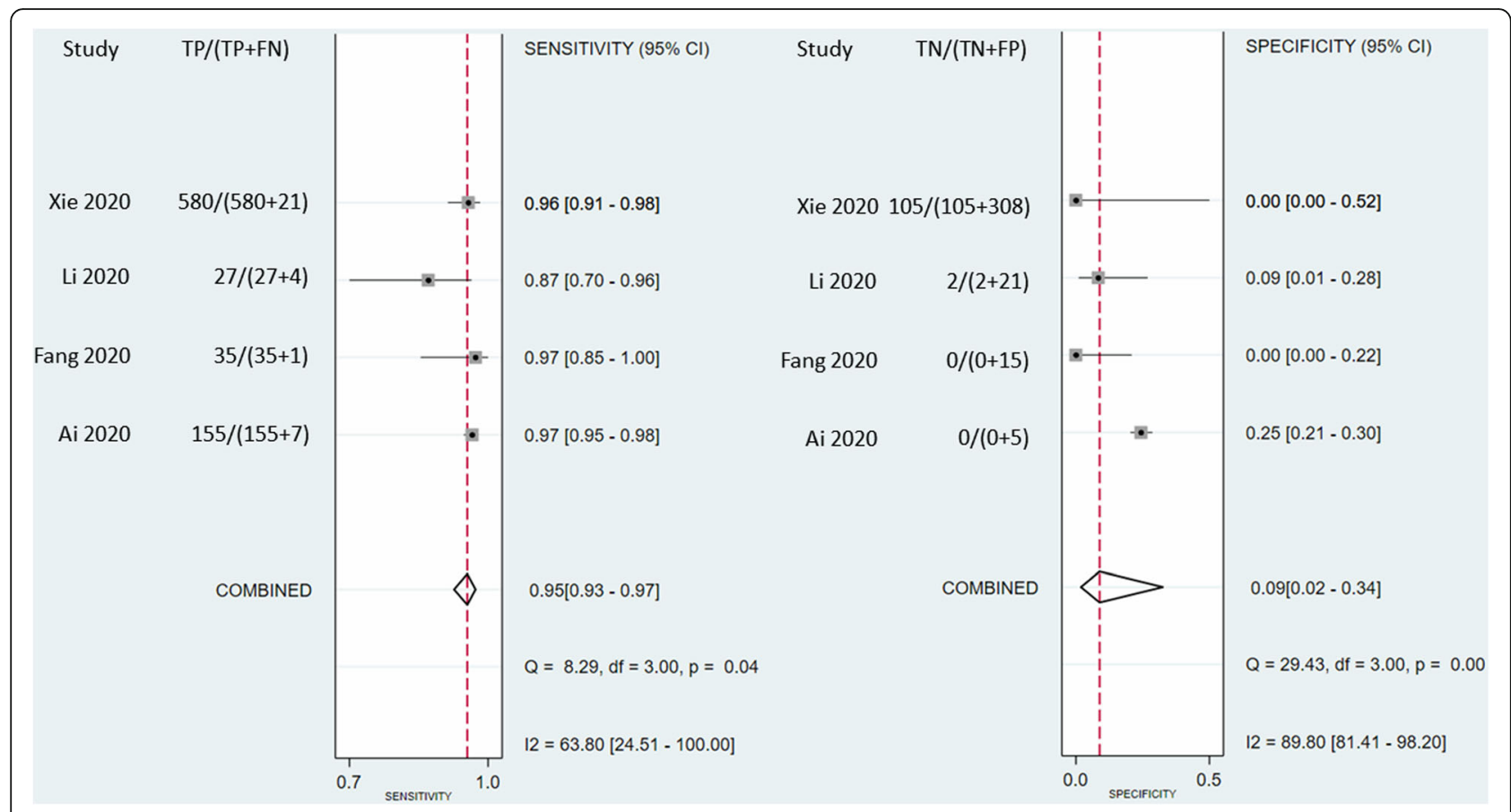

Fig. 1 Results of meta-analysis for the evaluation of the diagnostic value of chest CT in COVID-19. Study-specific and mean of sensitivity and specificity are presented in the Forest plots. TF, true positive; FN, false negative; TN, true negative; FP, false positive; Cl, confidence interval

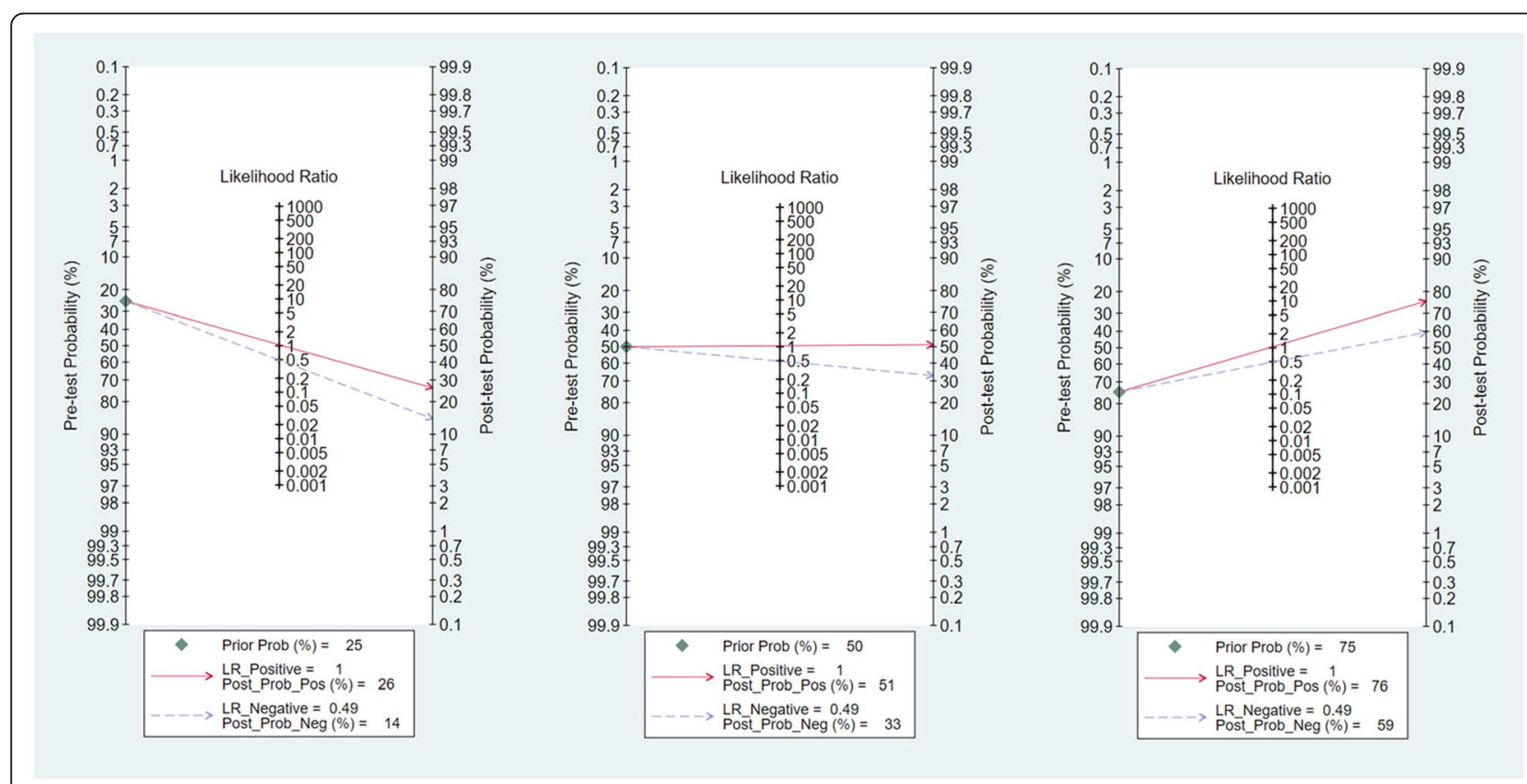

Fig. 2 Evaluating the clinical utility of chest CT for COVID-19 detection by Fagan's Nomogram plot. LR, likelihood ratio; prob, probability; pos, positive; neg, negative 
regarding low levels of specificity and likelihood ratio did not support the routine use of chest CT for COVID19 screening in suspicious patients. Our results from Fagan's Nomogram analyses suggested very little diagnostic value of using chest $\mathrm{CT}$ as the primary tool for COVID-19. One shortcoming of using chest CT is that patients are exposed to unnecessary radiation. The other shortcoming is that CT-scanning may increase the risk of nosocomial infection due to potential contamination of the environment.

Some limitations of our results should be mentioned. For example, whether radiologists were blind to other clinical data when interpreted CT or whether samples were adequately collected may influence the pooled results. Besides, we observed a high heterogeneity of both sensitivity and specificity as well as a wide range of specificity. The certainty of evidence, if graded, may be very low.

In conclusion, our pooled meta-analytic results of high sensitivity but poor specificity limit the routine use of chest CT as a primary tool for COVID-19 detection. Chest CT should only be arranged for individuals with certain clinical features in conjunction with RT-PCR tests. Further rigorous studies are required to find further refinements of our findings.

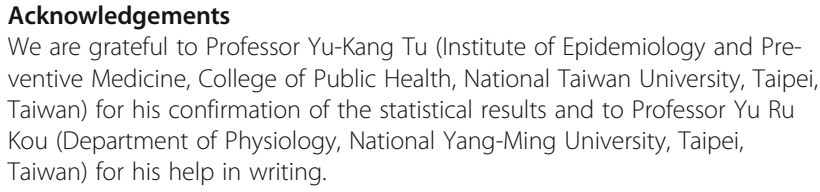
ventive Medicine, College of Public Health, National Taiwan University, Taipei, Taiwan) for his confirmation of the statistical results and to Professor Yu Ru Kou (Department of Physiology, National Yang-Ming University, Taipei, Taiwan) for his help in writing.

\section{Authors' contributions}

Edward PC Huang and CW Sung: critical analysis, interpretation of the data, and drafting of the manuscript. CH Chen: database search and data extraction. CY Fan: double confirmation of the enrolled studies and data. PC Lai: drafting of the manuscript. YT Huang: concept of the meta-analysis, statistical analyses, and drafting of the manuscript. The authors read and approved the final manuscript.

\section{Funding}

TCF-MP 108-01-02 from Buddhist Tzu Chi Medical Foundation Academic Advancement, Hualien, Taiwan.

\section{Availability of data and materials}

Not applicable.

\section{Ethics approval and consent to participate}

Not applicable. All authors of the manuscript have read and agreed to its content and are accountable for all aspects of the accuracy and integrity of the manuscript in accordance with ICMJE criteria.

\section{Consent for publication}

The article is original, has not already been published in a journal, and is not currently under consideration by another journal.

\section{Competing interests}

None in all authors.

\section{Author details}

${ }^{1}$ Department of Emergency Medicine, National Taiwan University Medical College and Hospital, Taipei, Taiwan. ${ }^{2}$ Department of Emergency Medicine,
National Taiwan University Hospital Hsin-Chu Branch, Hsinchu, Taiwan. ${ }^{3}$ Department of Medicine Education, National Taiwan University Hospital, Taipei, Taiwan. ${ }^{4}$ Evidence-based Medicine Center, Department of Medical Education, Hualien Tzu Chi Hospital, Buddhist Tzu Chi Medical Foundation, 707, Sec. 3, Chung-Yang Rd, Hualien 970, Taiwan. ${ }^{5}$ Department of Pediatrics, Hualien Tzu Chi Hospital, Buddhist Tzu Chi Medical Foundation, 707, Sec. 3. Chung-Yang Rd, Hualien 970, Taiwan. ${ }^{6}$ School of Medicine, Tzu Chi

University, Hualien, Taiwan. ${ }^{7}$ Division of Experimental Surgery, Department of Surgery, Hualien Tzu Chi Hospital, Buddhist Tzu Chi Medical Foundation, 707, Sec. 3, Chung-Yang Rd, Hualien 970, Taiwan. ${ }^{8}$ Surgical Intensive Care Unit, Department of Surgery, Hualien Tzu Chi Hospital, Buddhist Tzu Chi Medical Foundation, 707, Sec. 3, Chung-Yang Rd, Hualien 970, Taiwan. ${ }^{9}$ Department of Pharmacology, Tzu Chi University, Hualien, Taiwan.

Received: 30 March 2020 Accepted: 17 April 2020

Published online: 06 May 2020

References

1. World Health Organization. WHO Director-General's opening remarks at the media briefing on COVID-19 - 11 March 2020. https://www.who.int/dg/ speeches/detail/who-director-general-s-opening-remarks-at-the-mediabriefing-on-covid-19\%2D\%2D-11-march-2020. Accessed 13 May 2020

2. Ai T, Yang Z, Hou H, Zhan C, Chen C, Lv W, et al. Correlation of chest CT and RT-PCR testing in coronavirus disease 2019 (COVID-19) in China: a report of 1014 cases. Radiology. 2020;200642. https://doi.org/10.1148/radiol. 2020200642.

3. American College of Radiology. ACR Recommendations for the use of chest radiography and computed tomography (CT) for suspected COVID-19 infection. https://www.acr.org/Advocacy-and-Economics/ACR-PositionStatements/Recommendations-for-Chest-Radiography-and-CT-forSuspected-COVID19-Infection. Accessed 13 May 2020.

4. Xie X, Zhong Z, Zhao W, Zheng C, Wang F, Liu J. Chest CT for typical 2019nCoV pneumonia: relationship to negative RT-PCR testing. Radiology. 2020; 200343. https://doi.org/10.1148/radiol.2020200343.

5. Li YY, Wang WN, Lei Y, Zhang B, Yang J, Hu JW, et al. Comparison of the clinical characteristics between RNA positive and negative patients clinically diagnosed with 2019 novel coronavirus pneumonia. Zhonghua Jie $\mathrm{He} \mathrm{He}$ Hu Xi Za Zhi. 2020;43(0):E023. https://doi.org/10.3760/cma.j.cn11214720200214-00095.

6. Fang $Y$, Zhang $H$, Xie J, Lin M, Ying L, Pang P, et al. Sensitivity of chest CT for COVID-19: comparison to RT-PCR. Radiology. 2020;200432. https://doi. org/10.1148/radiol.2020200432

\section{Publisher's Note}

Springer Nature remains neutral with regard to jurisdictional claims in published maps and institutional affiliations.

Ready to submit your research? Choose BMC and benefit from

- fast, convenient online submission

- thorough peer review by experienced researchers in your field

- rapid publication on acceptance

- support for research data, including large and complex data types

- gold Open Access which fosters wider collaboration and increased citations

- maximum visibility for your research: over $100 \mathrm{M}$ website views per year

At $\mathrm{BMC}$, research is always in progress.

Learn more biomedcentral.com/submissions 